\title{
On-chip frequency-domain submillimeter-wave transceiver
}

\author{
N. Zamdmer and Qing $\mathrm{Hu}^{\mathrm{a})}$ \\ Department of Electrical Engineering and Computer Science and Research Laboratory of Electronics, \\ Massachusetts Institute of Technology, Cambridge, Massachusetts 02139
}

K. A. McIntosh and S. Verghese

Lincoln Laboratory, Massachusetts Institute of Technology, Lexington, Massachusetts 02420-9108

A. Förster
Institut für Schicht-und Ionentechnik (ISI), Forschungszentrum Jülich, Jülich D-52428, Germany

(Received 13 August 1999; accepted for publication 20 October 1999)

We demonstrate and model an on-chip frequency-domain transceiver which uses low-temperature-grown GaAs photoconductors to emit and detect continuous waves in the frequency range from 20 to $700 \mathrm{GHz}$ propagating in a coplanar waveguide circuit. Our device has a possible frequency resolution $(\sim 1 \mathrm{MHz})$ that is about $10^{3}$ times better than similar devices used for time-domain spectroscopy, and is therefore appropriate for high-resolution spectroscopy with a "lab-on-a-chip" -type integrated circuit. (c) 1999 American Institute of Physics.

[S0003-6951(99)01650-2]

Photoconductive emitters and receivers are attractive as components of submillimeter-wave spectroscopy systems because of their tunability, compactness, and ability to be monolithically integrated with antennas, transmission lines, and microelectronic devices. Such systems can be classified in either of two ways: as time-domain or frequency-domain systems, or as systems involving free-space or on-chip submillimeter-wave propagation. Time-domain systems, which contain a photoconductive pulse emitter and sampler excited by a mode-locked laser, are the most investigated. They have been used for free-space characterization of semiconductor materials, ${ }^{1}$ and on-chip characterization of ultrafast devices and circuits with 2.7 ps time resolution. ${ }^{2}$ The frequency resolution is the inverse of the time span over which the propagating pulse is sampled. This span is determined by the length of an optical delay line, which usually results in a frequency resolution greater than $1 \mathrm{GHz}^{2}$

A frequency-domain spectrometer was recently introduced $^{3}$ with a frequency resolution of better than 1 $\mathrm{MHz},{ }^{4}$ which is adequate for molecular line spectroscopy. ${ }^{4,5}$ Its source is a photomixer, which is a voltage-biased antenna-coupled photoconductor that generates a cw output at the difference frequency of the two tunable diode lasers that illuminate it. ${ }^{6}$ A photomixer is tunable from dc to about $5 \mathrm{THz},{ }^{6}$ and its linewidth is determined by the stability of the lasers that drive it. The spectrometer's detector is another antenna-coupled photoconductor illuminated by a delayed portion of the two overlapping laser beams that illuminate the source. The light incident on the detector creates a modulated photocarrier density that performs homodyne detection of the continuous wave from the source. ${ }^{7}$

In this letter, we describe the on-chip version of the above frequency-domain spectrometer. Such a spectrometer is attractive because it has the frequency resolution required for low-pressure gas spectroscopy. It is also compact and

${ }^{a)}$ Electronic mail: qhu@mit.edu inexpensive, and it can be part of a microfluidic, "lab-on-achip" -type circuit. Furthermore, it has the advantage over a free-space spectrometer that it can be used to test microelectronic devices without the collimation and focusing of submillimeter waves. As the first step in the development of an on-chip frequency-domain spectrometer, here we investigate the performance of an on-chip transceiver containing only uninterrupted coplanar waveguides (CPWs).

Our circuit, shown in Fig. 1(a), is the same as the one on which we performed time-domain experiments. ${ }^{8}$ The circuit

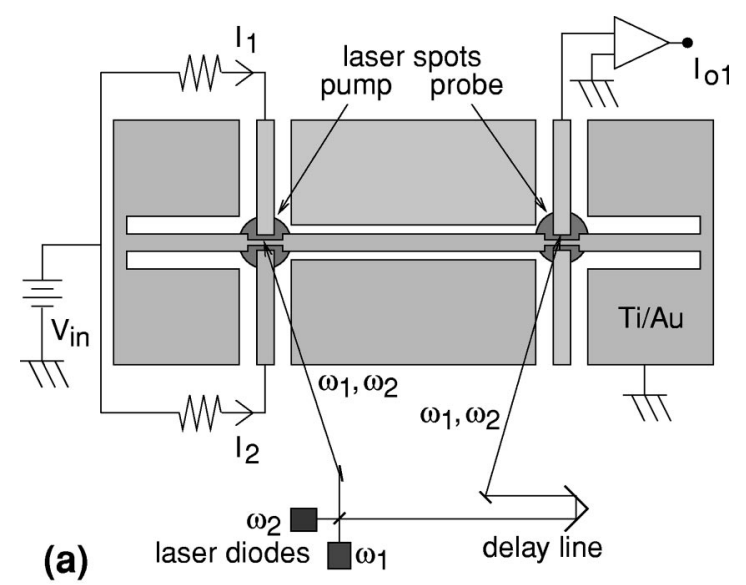

(b)

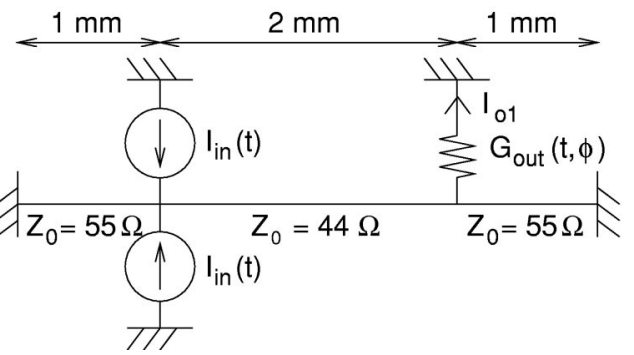

FIG. 1. (a) Diagram of the experimental circuit, showing its electrical bias and optical input. (b) Microwave circuit model of the experimental circuit. 
has a biased pump photoconductor and an unbiased probe photoconductor connected by a main CPW, and other parasitic CPWs which provide dc electrical contact to the photoconductors. As illustrated in Fig. 1(a), we excited propagating electromagnetic waves at the pump by illuminating the pump photoconductor with two overlapping diode laser beams with a difference frequency $f=\left|\omega_{2}-\omega_{1}\right| / 2 \pi$. We performed homodyne detection of those waves by illuminating the probe with a delayed portion of the same laser beams. We describe the relative delay between the pump and probe beams with the phase $\phi$, which is defined as $\phi=2 \pi f\left(z_{\text {probe }}\right.$ $\left.-z_{\text {pump }}\right) / c$, where $c$ is the speed of light and $z_{\text {probe }}$ and $z_{\text {pump }}$ are the path lengths of the probe and pump beams, respectively. $z_{\text {pump }}$ includes the electrical length of the 2-mm-long CPW between the pump and probe photoconductors. We expect the output dc current $I_{o 1}$, collected with a transimpedance amplifier, to vary sinusoidally with $\phi$ because of the homodyne detection performed at the probe photoconductor. We describe this dependence as $I_{o 1}(\phi)=I_{0} \cos (\phi+\delta)$, where $I_{0}$ is the sinusoid's amplitude. The argument of cosine contains two terms: the phase $\phi$, which is due only to the path lengths of the pump and probe beams; and the phase $\delta$, which describes the response of the circuit and any device or specimen inserted in it. For example, $\delta$ may be nonzero because of the dispersion of the CPWs or circuit resonance. Our aim was to measure $I_{0}$ and $\delta$ as functions of $f$. Together, $I_{0}$ and $\delta$ contain all the information necessary for coherent spectroscopy.

The CPW circuit is composed of a single layer of patterned $\mathrm{Ti} / \mathrm{Au}$ on a photoconductive low-temperature-grown (LTG) GaAs epitaxial layer. The LTG GaAs has a carrier lifetime of $1.1 \mathrm{ps}$, and the other characteristics and dimensions of the circuit are described in Ref. 8. The two photoconductors each have two active regions so that they can selectively generate and detect a CPW's two quasitransverse electromagnetic (TEM) propagating modes. ${ }^{8}$ We biased and illuminated the two active regions of the pump photoconductor equally to generate only the mode with odd transverse electric field, which is less dispersive and attenuative than the even mode. ${ }^{8}$ We achieved equal illumination by steering the pump beam to equalize the two dc pump photocurrents $I_{1}$ and $I_{2}$ at a given bias $V_{\text {in }}$. There is no need to align the probe beam to maintain mode purity, because the peak photoconductance $(\sim 1 \mathrm{M} \Omega)$ of the probe is much less than the characteristic impedance $Z_{0}$ of the CPWs $(\sim 50 \Omega)$, and thus, does not affect wave propagation. We aligned the probe beam to maximize the dc photoconductance of only one side of the probe photoconductor, so as to maximize the singlesided output $I_{o 1}$.

We measured the sinusoidally varying $I_{o 1}(\phi)$ at ten discrete values of $\phi$ within one cycle for every $f$ of interest. We controlled $\phi$ by stepping a computer-controlled translation stage of the optical delay line [see Fig. 1(a)]. $I_{o 1}$ contains a large $\phi$-independent background which must be rejected in order to measure the $\phi$-dependent portion with high signalto-noise ratio. We accomplished this rejection by dithering a speaker-mounted retroreflector in the optical delay line at 24 Hz. This modulates $z_{\text {probe }}$, and therefore $\phi$, and we detected the consequent modulation of $I_{o 1}$ with a lock-in amplifier, essentially measuring $d I_{o 1} / d \phi$. At every $f$, we adjusted the Downloaded 15 Dec 2006 to 134.94.122.39. Redistribution subject
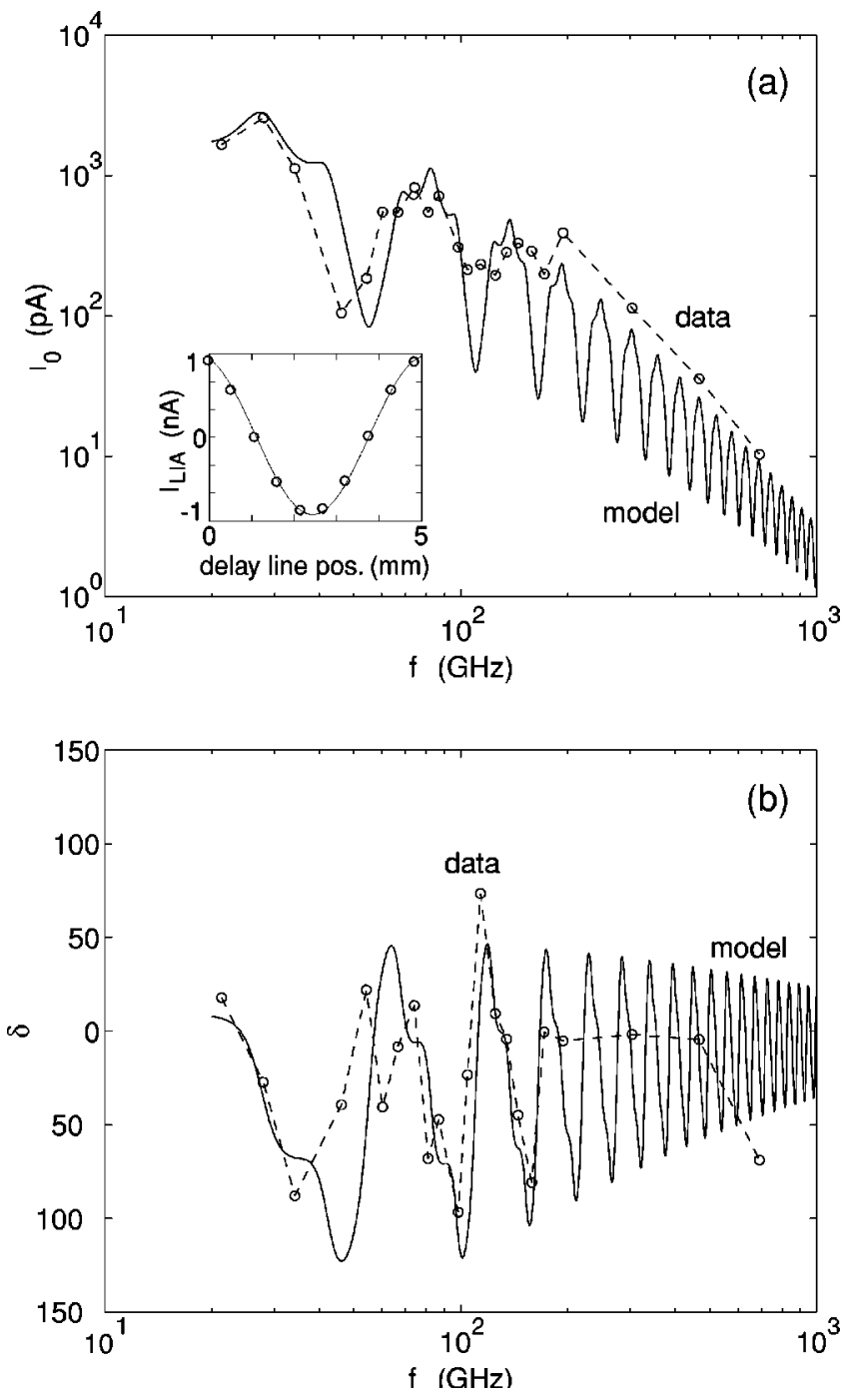

FIG. 2. Measured data and model of the amplitude and phase spectra (a) $I_{0}(f)$ and (b) $\delta(f)$. Inset: output of lock-in amplifier $I_{\text {LIA }}$ vs delay line position at $f=27.9 \mathrm{GHz}$, compared to a best-fit sinusoid.

speaker throw to maximize the lock-in amplifier's output $I_{\text {LIA }}(\phi)$. To extract $I_{0}$ from $I_{\text {LIA }}(\phi)$, we assume that the speaker moves sinusoidally in space, and therefore, the amplitude of $I_{\mathrm{LIA}}(\phi)$ is $0.58 I_{0}$, where 0.58 is the maximum value of $J_{1}$, the first-order Bessel function of the first kind.

Because the length $z_{\text {pump }}-z_{\text {probe }}$ was not known exactly, the phase $\delta$ could not be extracted from any one measurement of $I_{\mathrm{LIA}}(\phi)$ at a single frequency. We derived the spectrum $\delta(f)$ from our set of measurements over all $f$ by finding the value of $z_{\text {pump }}-z_{\text {probe }}$ for which the resulting spectrum $\delta(f)$ corresponded best with theory. Since we took data at 22 different $f$ over a large frequency range $(21.3-689 \mathrm{GHz})$, there is little ambiguity in the derived $\delta(f)$.

We performed all measurements with $63.7 \mathrm{~mW}$ of total average optical power incident on the pump photoconductor and $38.7 \mathrm{~mW}$ incident on the probe photoconductor, with both laser diodes operating near $850 \mathrm{~nm}$. We varied $f$ by tuning the laser temperatures. The pump bias $V_{\text {in }}$ was $30 \mathrm{~V}$, and $I_{1}$ and $I_{2}$ were both $53 \mu \mathrm{A}$. A typical cycle of measured data $I_{\text {LIA }}(\phi)$ is shown in the inset of Fig. 2(a), which fits a sinusoid very well. The measured spectra $I_{0}(f)$ and $\delta(f)$ are shown in Fig. 2.

The most prominent features of the data are the oscilla- 
tions in $I_{0}(f)$ and $\delta(f)$, the roll-off in $I_{0}(f)$, and the nonzero negative average value of $\delta(f)$. The oscillations are accurately modeled as resonances due to reflections at the shorts that terminate the parasitic CPWs that contact the center conductor of the main CPW. Indeed, our previously published time-domain data show that these shorts are strongly reflective. ${ }^{8}$ The roll-off in amplitude of the output is due to the finite response time $\tau$ of the photoconductors. This rolloff is common to all systems containing photoconductors.

The negative average value of $\delta(f)$ is probably caused by a difference in the response times of the pump and probe photoconductors. For $f$ greater than $1 / 2 \pi \tau$, the modulation of photocarriers lags behind the modulation of the optical input. If the response times of the pump and probe photoconductors were identical, then these phase lags would cancel at all $f$, and $\delta$ would be zero, apart from the contributions due to circuit resonance. However, the pump and probe photoconductors are not identical, because the pump is at high bias and the probe is unbiased. Our studies show that biased LTG GaAs photoconductors have a slower response time than unbiased ones due to field-induced reduction of the electron capture cross section of recombination centers. ${ }^{9}$ This causes $\delta$ to be negative for frequencies between $1 / 2 \pi \tau_{\text {pump }}$ and $1 / 2 \pi \tau_{\text {probe }}$, in agreement with our data, where $\tau_{\text {pump }}$ and $\tau_{\text {probe }}$ are, respectively, the pump and probe photoconductor response times. We confirmed that the pump bias $V_{\text {in }}$ does indeed influence $\delta$ by observing $\delta$ to increase with a decreasing $V_{\text {in }}$ at a fixed $f$ of $85 \mathrm{GHz}$.

We fit the measured spectra to a model based on the circuit shown in Fig. 1(b). The two active regions of the pump photoconductor are modeled as current sources

$$
I_{\text {in }}(t)=I_{1}\left[1+\operatorname{Re}\left(\frac{\exp (j \omega t)}{1+j \omega \tau_{\text {pump }}}\right)\right],
$$

where $\omega=2 \pi f$. Similarly, the single utilized active region of the probe photoconductor is modeled as the time-varying conductance

$$
G_{\text {out }}(t, \phi)=G_{1}\left[1+\operatorname{Re}\left(\frac{\exp (j \omega t-j \phi)}{1+j \omega \tau_{\text {probe }}}\right)\right],
$$

where $G_{1}$ is the dc photoconductance of the probe photoconductor, which we measured to be $1.26 \times 10^{-6} \Omega^{-1}$. We assume that the two laser diode beams were perfectly overlapped, and thus the ac and dc components of $I_{\mathrm{in}}(t)$ and $G_{\text {out }}(t)$ have equal magnitude at low frequencies. We also assume that the CPWs have a propagation constant $\gamma$ $=\alpha(f)+j 2 \pi f / \mathrm{v}_{p}$, where $\alpha(f)$ is the attenuation constant to be fit to the data, and $\mathrm{v}_{p}$ is the phase velocity of a coplanar transmission line on a semi-infinite GaAs substrate. We neglect dispersion and assume $\mathrm{V}_{p}$ to be the frequencyindependent value $0.37 c$, or $1.1 \times 10^{8} \mathrm{~m} / \mathrm{s} .{ }^{10}$ We use standard microwave circuit analysis to calculate $I_{o 1}$, the $\phi$-dependent dc current generated at the probe. We assume that only the horizontal CPWs of Fig. 1(a) form the ac circuit, as time- domain measurements indicate that the vertical CPWs have a negligible impact. ${ }^{8}$ The values of the characteristic impedances of the CPWs in the circuit are shown in Fig. 1(b).

Our model was fit to the data with fitting parameters $\alpha(f)$ and $\tau_{\text {pump }} . \tau_{\text {probe }}$ is the zero-field value mentioned earlier, 1.1 ps. $\alpha(f)$ was modeled as a constant $\alpha_{\mathrm{dc}}$ for $f$ $<f_{\text {skin }}$, and as proportional to $f^{1 / 2}$ for $f>f_{\text {skin }}$, where $f_{\text {skin }}$ is the frequency above which the skin effect becomes important. $\alpha_{\mathrm{dc}}$ and $f_{\text {skin }}$ were treated as fitting parameters. The fit shown in Fig. 2 was obtained with the fitting parameter values $\tau_{\text {pump }}=5.3 \mathrm{ps}, \alpha_{\mathrm{dc}}=0.2 \mathrm{~mm}^{-1}$, and $f_{\text {skin }}=100 \mathrm{GHz}$. The value of $\tau_{\text {pump }}$ is in agreement with the model discussed in Ref. 9, and provides a confirmation of that model. The values of $\alpha_{\mathrm{dc}}$ and $f_{\text {skin }}$ are also reasonable considering the conductivity and dimensions of the CPW metallization. The fit is quite good, except for the valleys of $I_{0}(f)$, where the detected signal is small. The apparent offset between the data and model near $f=50 \mathrm{GHz}$ may be due to dispersion of the quasitransverse electromagnetic (TEM) modes of the CPWs at microwave frequencies, as observed by others. ${ }^{2}$

In summary, we have performed measurements on an on-chip frequency-domain transceiver over a broad range of the millimeter- and submillimeter-wave spectrum (21.3-689 $\mathrm{GHz})$ with unprecedented frequency resolution $(\sim 1 \mathrm{MHz}$ possible). Such measurements are a step in the development of inexpensive, compact on-chip spectrometers that are potentially useful for chemical and biological sensing, as well as for characterization of high-frequency electronic devices.

This work was supported at MIT by the National Science Foundation, and at MIT Lincoln Laboratory by the National Aeronautics and Space Administration, Office of Space Access and Technology, through the Center for Space Microelectronics Technology, Jet Propulsion Laboratory, California Institute of Technology.

${ }^{1}$ M. C. Nuss and J. Orenstein, in Millimeter and Submillimeter Spectroscopy of Solids, edited by G. Grüner (Springer, Berlin, 1998).

${ }^{2}$ T. Pfeifer, H.-M. Heiliger, T. Löffler, C. Ohlhoff, C. Meyer, G. Lüpke, H. G. Roskos, and H. Kurz, IEEE J. Sel. Top. Quantum Electron. 2, 586 (1996).

${ }^{3}$ S. Verghese, K. A. McIntosh, S. Calawa, W. F. Dinatale, and E. K. Duerr, Appl. Phys. Lett. 73, 3824 (1998).

${ }^{4}$ P. Chen, G. A. Blake, M. C. Gaidis, E. R. Brown, K. A. McIntosh, S. Y. Chou, M. I. Nathan, and F. Williamson, Appl. Phys. Lett. 71, 1601 (1997).

${ }^{5}$ A. S. Pine, R. D. Suenram, E. R. Brown, and K. A. McIntosh, J. Mol. Spectrosc. 175, 37 (1996).

${ }^{6}$ K. A. McIntosh, E. R. Brown, K. B. Nichols, O. B. McMahon, W. F. Dinatale, and T. M. Lyszczarz, Appl. Phys. Lett. 67, 3844 (1995).

${ }^{7}$ Another form of high-frequency-resolution photoconductive spectroscopy is described in T. M. Goyette, W. Guo, F. C De Lucia, J. C. Swartz, H. O. Everitt, B. D. Guenther, and E. R. Brown, Appl. Phys. Lett. 67, 3810 (1995).

${ }^{8}$ N. Zamdmer, Q. Hu, S. Verghese, and A. Förster, Appl. Phys. Lett. 74, 1039 (1999).

${ }^{9}$ N. Zamdmer, Q. Hu, K. A. McIntosh, and S. Verghese, Appl. Phys. Lett. 75, 2313 (1999).

${ }^{10}$ J. Allam, N. de B. Baynes, J. R. A. Cleaver, K. Ogawa, T. Mishima, and I. Ohbu, Opt. Quantum Electron. 28, 875 (1996). 